\title{
TeV $\gamma$-ray light curve and energy spectrum of Mkn 421 during its 2001 flare as measured with HEGRA CT1 ${ }^{\star}$
}

F. Aharonian ${ }^{1}$, A. Akhperjanian ${ }^{7}$, M. Beilicke ${ }^{4}$, K. Bernlöhr ${ }^{1}$, H. G. Börst ${ }^{5}$, H. Bojahr ${ }^{6}$, O. Bolz ${ }^{1}$, J. A. Coarasa ${ }^{2}$, J. L. Contreras ${ }^{3}$, J. Cortina ${ }^{2,8}$, S. Denninghoff ${ }^{2}$, V. Fonseca ${ }^{3}$, M. Girma ${ }^{1}$, F. Goebel ${ }^{2}$, N. Götting ${ }^{4}$, G. Heinzelmann ${ }^{4}$, G. Hermann ${ }^{1}$, A. Heusler ${ }^{1}$, W. Hofmann ${ }^{1}$, D. Horns ${ }^{1}$, I. Jung ${ }^{1}$, R. Kankanyan ${ }^{1}$, M. Kestel ${ }^{2}$, J. Kettler ${ }^{1}$, A. Kohnle ${ }^{1}$, A. Konopelko ${ }^{1}$, D. Kranich ${ }^{2}$, H. Krawczynski ${ }^{1,10}$, H. Lampeitl ${ }^{4}$, M. López ${ }^{3}$, E. Lorenz ${ }^{2}$, F. Lucarelli ${ }^{3}$, O. Mang ${ }^{5}$, H. Meyer ${ }^{6}$, R. Mirzoyan ${ }^{2}$, A. Moralejo ${ }^{3}$, E. Oña-Wilhelmi ${ }^{3}$, D. Paneque ${ }^{2}$, M. Panter $^{1}$, A. Plyasheshnikov ${ }^{1,7}$, G. Pühlhofer ${ }^{1}$, R. de los Reyes ${ }^{3}$, W. Rhode ${ }^{6}$, J. Ripken ${ }^{4}$, G. Rowell ${ }^{1}$, V. Sahakian ${ }^{7}$, M. Samorski ${ }^{5}$, M. Schilling ${ }^{5}$, T. Schweizer ${ }^{8}$, I. Sevilla ${ }^{3}$, M. Siems ${ }^{5}$, D. Sobczyńska ${ }^{9}$, W. Stamm ${ }^{5}$, M. Tluczykont ${ }^{4}$, N. Tonello ${ }^{2}$, V. Vitale ${ }^{2}$, H. J. Völk ${ }^{1}$, R. M. Wagner ${ }^{2}$, C. A. Wiedner ${ }^{1}$, and W. Wittek ${ }^{2}$

\footnotetext{
1 Max-Planck-Institut für Kernphysik, Postfach 103980, 69029 Heidelberg, Germany

2 Max-Planck-Institut für Physik, Föhringer Ring 6, 80805 München, Germany

3 Universidad Complutense, Facultad de Ciencias Físicas, Ciudad Universitaria, 28040 Madrid, Spain

${ }^{4}$ Universität Hamburg, Institut für Experimentalphysik, Luruper Chaussee 149, 22761 Hamburg, Germany

5 Universität Kiel, Institut für Experimentelle und Angewandte Physik, Leibnizstraße 15-19, 24118 Kiel, Germany

${ }^{6}$ Universität Wuppertal, Fachbereich Physik, Gaußstr. 20, 42097 Wuppertal, Germany

7 Yerevan Physics Institute, Alikhanian Br. 2, 375036 Yerevan, Armenia

${ }^{8}$ Institut de Física d'Altes Energies, Universitat Autònoma de Barcelona, 08193 Bellaterra, Spain

9 Experimental Physics Dept., University of Łódź ul. Pomorska 149/153, 90-236 Łódź, Poland

${ }^{10}$ Now at Yale University, PO Box 208101, New Haven, CT 06520-8101, USA
}

Received 12 March 2003 / Accepted 7 August 2003

\begin{abstract}
In the first months of 2001 the AGN Mkn 421 showed highly variable, strong TeV activity at flux levels frequently exceeding 1 Crab. Here we present the light curve and energy spectrum of Mkn 421 as measured with the HEGRA stand alone telescope CT1. Around 30\% of the data were taken under moonlight conditions. The spectrum shows a significant exponential energy cutoff at around 3.4 TeV. The results from the dark night- and the moon data are in excellent agreement with each other. A significant spectral shape variation depending on the flux level has been found. The TeV light curve is also found to be highly correlated with the X-ray light curve of the RXTE / ASM satellite, showing no significant time lag larger than $0.2 \mathrm{~d}$. The derived correlation coefficient of 0.83 corresponds to a $5.2 \sigma$ significance.
\end{abstract}

Key words. gamma rays: observations - BL Lacertae objects: individual: Mkn 421

\section{Introduction}

Mkn 421 is the closest known TeV blazar $(z=0.030)$ and was the first extragalactic source found to emit $\gamma$-rays in the TeV energy range using Imaging Air Cherenkov Telescopes (IACTs) (Punch et al. 1992, confirmed by HEGRA in Petry et al. 1996). Mkn 421 is the source with the fastest observed flux variations reported from $\mathrm{TeV} \gamma$-ray emitters, with an occasionally flux doubling time of 15 min (Gaidos et al. 1996; Aharonian et al. 2002).

Send offprint requests to: D. Kranich, J. Cortina, e-mail: daniel@mppmu.mpg.de; cortina@ifae.es

* Table 4 is only available in electronic form at the CDS via anonymous ftp to cdsarc.u-strasbg.fr $(130.79 .128 .5)$ or via http://cdsweb.u-strasbg.fr/cgi-bin/qcat?J/A+A/410/813
Mkn 501, another TeV blazar at a similar redshift $(z=$ 0.034), shows TeV emission with a cutoff around $6 \mathrm{TeV}$ in its energy spectrum from 1997 (Samuelson et al. 1998; Aharonian et al. 1999a; Djannati-Ata1 et al. 1999). This cutoff might either be intrinsic to the source, caused by the absorption on photons near the source (Dermer \& Schlickeiser 1994) or caused by an absorption process due to the interaction with the up to now poorly-quantified extragalactic background light (EBL) (Gould \& Schréder 1966; Stecker et al. 1992), i.e. TeV $\gamma$-radiation might provide an efficient probe of the EBL around $10 \mu \mathrm{m}$ wavelength (Stecker \& de Jager 1993). While a high significance observation of an unbroken power law spectrum of Mkn 421 would indicate that the cutoff of Mkn 501 is source inherent, the observation of a cutoff in the Mkn 421 spectrum at about the same energy would strengthen the case of a sizeable photon density of the EBL. Therefore the detailed study of 
the TeV spectra of both Mkn 501 and Mkn 421 is of particular interest for cosmology.

In January 2001, after an alert from the Whipple collaboration, strong and highly variable $\mathrm{TeV}$ and $\mathrm{X}$-ray emission of Mkn 421 was reported by HEGRA and RXTE (Börst et al. 2001). The source remained very active until April 2001 and was observed by several groups during that time (Aharonian et al. 2002; Krennrich et al. 2002; Okumura et al. 2002). Here we describe the results of the analysis of the data taken during this period with the stand alone telescope HEGRA CT1 (Mirzoyan et al. 1994). A TeV light curve for the whole observation season is presented and compared with the RXTE AllSky Monitor (ASM) X-ray light curve in the $2-10 \mathrm{keV}$ energy range. The high statistics of the sample allows to extract an accurate energy spectrum and ascertain if there is a significant energy cutoff at $\mathrm{TeV}$ energies.

The results which are presented here are in good agreement with the results from an independent and completely different analysis of the same data (Schweizer 2002, Thesis, unpublished; Cortina et al. 2002).

\section{Observations and data analysis}

The stand alone IACT CT1 is part of the HEGRA detector located at the Roque de los Muchachos Observatory on the Canary Island La Palma, Spain $\left(28.75^{\circ} \mathrm{N}, 17.89^{\circ} \mathrm{W}\right.$, 2200 ma.s.1.). CT1 started operation in 1992 and was upgraded in 1994 with a high resolution camera, equipped with 127 pixels $^{1}$ of $0.25^{\circ}$ diameter. The FOV of the hexagonal camera has a minimum angular diameter of $\sim 3^{\circ}$. The original glass mirrors were replaced by all-aluminum mirrors and the reflector size has been enlarged in November 1997, making up to a total reflecting area of $10 \mathrm{~m}^{2}$. A next-neighbor trigger was implemented during the same year. Both improvements resulted in a reduction of the energy threshold down to $\approx 700 \mathrm{GeV}$ (Cortina et al. 2000). The trigger rate on cosmic ray events close to the zenith after a noise filter is now around $3.5 \mathrm{~Hz}$.

For this analysis a refined Monte Carlo simulation has been used (Sobczyńska \& Lorenz 2002). The most important improvement with respect to earlier simulations is that single photo electron pulses are now simulated with a variable pulse height and time shape according to the measured distributions. Also, the spread of the Cherenkov photon arrival time and the additional widening due to various detector elements was fully taken into account. Previously used simulations were based on simple photo electron counting within the trigger coincidence window. The resulting effective areas at lowest energies are smaller compared to those obtained with the previously used simulations. This is especially important close to the telescope threshold, where the old simulation overestimated the trigger efficiencies, leading to too low flux estimates in the first few energy bins of a derived energy spectrum ${ }^{2}$.

\footnotetext{
${ }^{1}$ A pixel consists of a light concentrator, a photo multiplier tube and a preamplifier.

${ }^{2}$ It should be noted, that this problem only affects CT1 data taken with the old detector setup, i.e. data taken before 1998. In addition the published results on the $1997 \mathrm{Mkn} 501$ data remain valid since
}

Table 1. The observation times (after quality cuts) of Mkn 421 with HEGRA CT1 in the 2001 observation period for the different HV settings, along with the significance of the signal and the number of candidate gamma events in each time period.

\begin{tabular}{cccc}
\hline \hline HV Setting & $\begin{array}{c}\text { Exposure } \\
(\mathrm{h})\end{array}$ & $\begin{array}{c}\text { Significance } \\
(\sigma)\end{array}$ & $\begin{array}{c}\text { Excess } \\
(\text { Events })\end{array}$ \\
\hline NOM & 262.7 & 101.9 & $13718 \pm 111$ \\
HV00 & 46.9 & 34.8 & $2398 \pm 56$ \\
HV04 & 23.0 & 19.9 & $1014 \pm 33$ \\
HV08 & 13.4 & 12.2 & $430 \pm 27$ \\
HV12 & 12.5 & 9.7 & $233 \pm 23$ \\
\hline
\end{tabular}

The photo multipliers of the telescope are operated under nominal high voltage (HV) during dark nights (Nominal setting: NOM) and for low moon illumination (HV00 setting) and with a number of reduced HV settings depending on the moon illumination and the angular distance between the moon and the observed object (HV04, HV08 and HV12 settings, respectively standing for $4 \%, 8 \%$ and $12 \%$ reduction of the nominal HV). The reduced HV allows for extended observation time coverage albeit with an increase of the energy threshold (Kranich et al. 1999).

The total observation time for the different HV settings is summarized in Table 1. Almost 300 hours were recorded during dark nights and about 96 hours under the presence of moonlight. The minimum zenith angle (ZA) for the observation of Mkn 421 is around $9^{\circ}$. The maximum ZA taken into account in the analysis is $60^{\circ}$. After image cleaning in total more than $3 \times 10^{6}$ events remained.

In order to select high quality data, a number of cuts were applied to the raw data:

- The cosmic ray rate as a function of ZA was required to be close to the nominal rate under the best atmospheric conditions.

- Nights with technical problems were rejected.

In total, $15 \mathrm{~h}$ of data were excluded due to technical problems and $21 \mathrm{~h}$ of data were excluded due to bad weather conditions and thus low cosmic ray rates.

In addition to the above mentioned quality cuts the socalled dynamical supercuts (Kranich 2001, Thesis, unpublished) are applied to the image parameters. These cuts vary with zenith angle, the image parameters SIZE (a rough measure of the shower energy) and DIST (a coarse measure of the impact parameter) and significantly enhance the $\gamma$ /hadron ratio. The dynamical supercuts (including a cut on the image parameter ALPHA) retain about $44 \%$ of the $\gamma$ showers while the hadron showers are suppressed by a factor 410 . Figure 1 shows the ALPHA distribution of the NOM data set after the dynamical supercuts. The significance of the signal and the number of excess events after dynamical supercuts for the individual data samples are also listed in Table 1.

either the effect has been taken into account (Kranich et al. 2001) or the threshold region was ignored in the energy spectrum determination (Aharonian et al. 1999a). 


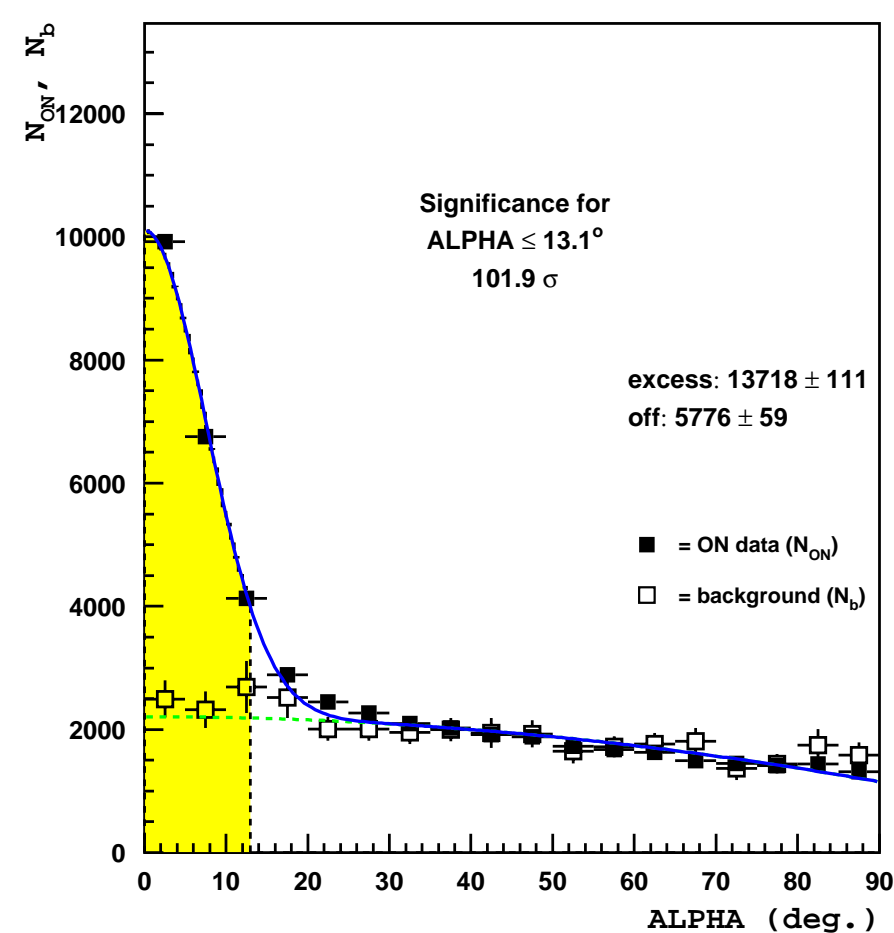

Fig. 1. The total signal of Mkn 421 using the $262.7 \mathrm{~h}$ of NOM data. The plot shows the On event (filled squares) and normalized Off event distribution (open squares, in total nearly 35000 events before normalization) as a function of the image parameter ALPHA. The shaded region denotes the source region, where most of the signal events show up in case of On-source observations. The solid and dashed line has been derived from a $\chi^{2}$-fit to the On-event distribution.

In order to counteract leakage effects in the relatively small $3^{\circ}$ diameter CT1 camera, a new empirical image parameter LEAKAGE has been introduced. It is defined as:

LEAKAGE $:=\sum_{\text {edge pixels }} q_{i} / \sum_{\text {all pixels }} q_{i}$

where $q_{i}$ is the light content in an individual pixel and the edge-pixels are the pixels located on the edge of the camera (Schweizer 2002, Thesis, unpublished). The LEAKAGE parameter allows to correct in part for the fraction of the shower signal lost outside the camera FOV and improves substantially the reconstruction of the shower image and the primary energy, particularly at high $\gamma$-ray energies above $5 \mathrm{TeV}$.

The procedure to derive 2-dimensional source maps from CT1 data is based on the method described in Lessard et al. (2001). In short, the method can be summarized as follows: the arrival direction for a given shower event is estimated as the point on the major axis of the shower image located at a distance DISP to the shower centroid. The DISP parameter is a function of the elongation of the shower image and is defined as DISP $:=\xi \cdot(1-$ WIDTH/LENGTH $)$ where $\xi$ is a scaling parameter. The final source map is build up from the arrival directions of all shower events. In the case of the CT1 data, the DISP parameter was slightly modified to include the LEAKAGE parameter:

DISP $:=\xi \cdot\left(1-\frac{\text { WIDTH }}{\mathrm{LENGTH} \cdot(1+\eta \cdot \mathrm{LEAKAGE})}\right)$.

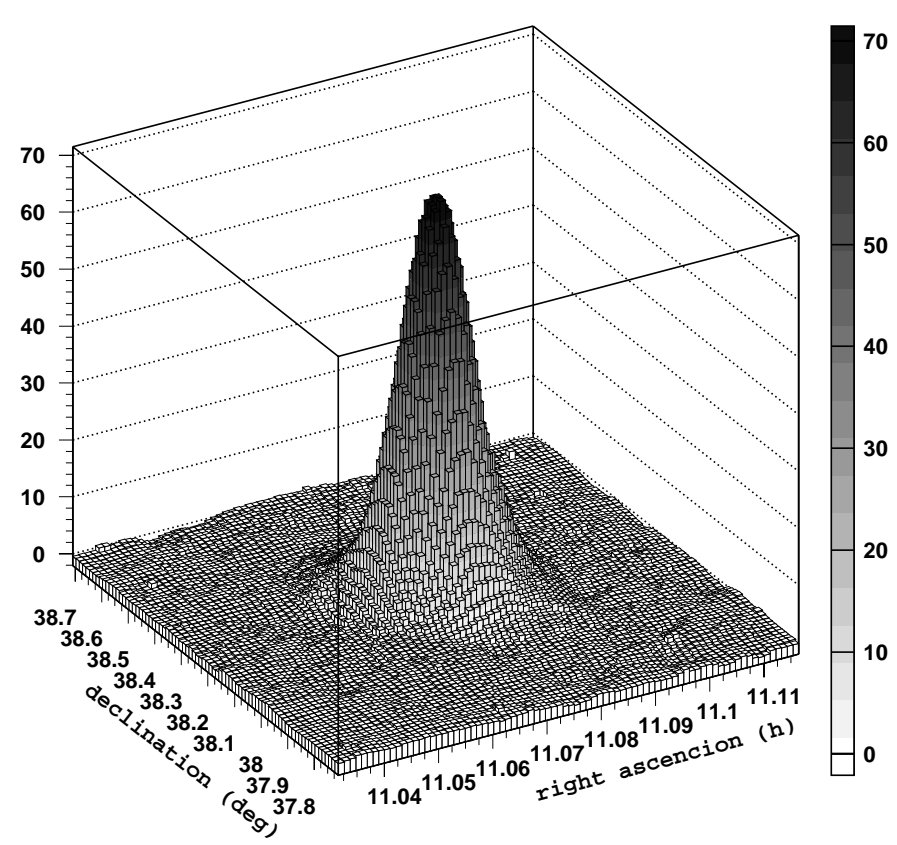

Fig. 2. Excess arrival directions for the NOM data set of Mkn 421. The actual source position is at RA $11.07 \mathrm{~h}$ and Dec 38.21 $1^{\circ}$ (Epoch J2000.0) and the estimated source position was found within $1^{\prime}$ distance.

The inclusion of the LEAKAGE parameter corrects for truncation effects of the LENGTH parameter. The free variables $\xi$ and $\eta$ were determined from Monte Carlo simulations as $\xi=$ 1.3 and $\eta=6.6$.

As a modification of this method we determine a set of possible arrival directions for each shower event (in the following called arrival distribution):

Taking into account the value and error of both the DISP parameter and the orientation of the major axis of each shower image one can calculate the most probable intersection point for any (randomly chosen) triple of shower images by means of a $\chi^{2}$ fit. The arrival distribution for a given shower event is then defined by the subset of all those intersection points, where the given shower event has been part of the triple ${ }^{3}$. Poorly defined intersection points with a $\chi^{2} /$ d.o.f. $>6 / 3$ were rejected. Each derived arrival distribution was then normalized to unity. The final source map is given by the superposition of arrival distributions from all individual shower events.

The basic idea of this method is that all intersection points of pure $\gamma$ shower triples should point towards the same sky region whereas a more or less isotropic distribution is expected whenever hadronic shower images are involved.

The distribution of arrival directions is determined for both OFF and ON data and the OFF data distribution is then normalized and subtracted from the $\mathrm{ON}$ data. Figure 2 shows the resulting distribution of excess arrival directions for the total Mkn 421 NOM data set. A fit of a two dimensional Gaussian distribution yields an angular resolution of $\sigma_{x, y} \simeq 0.08^{\circ}$.

\footnotetext{
${ }^{3}$ Due to the large computational expense of this method the number of intersection points per shower event have been limited to 500 .
} 
Mkn 421 - Lightcurve 2000-01
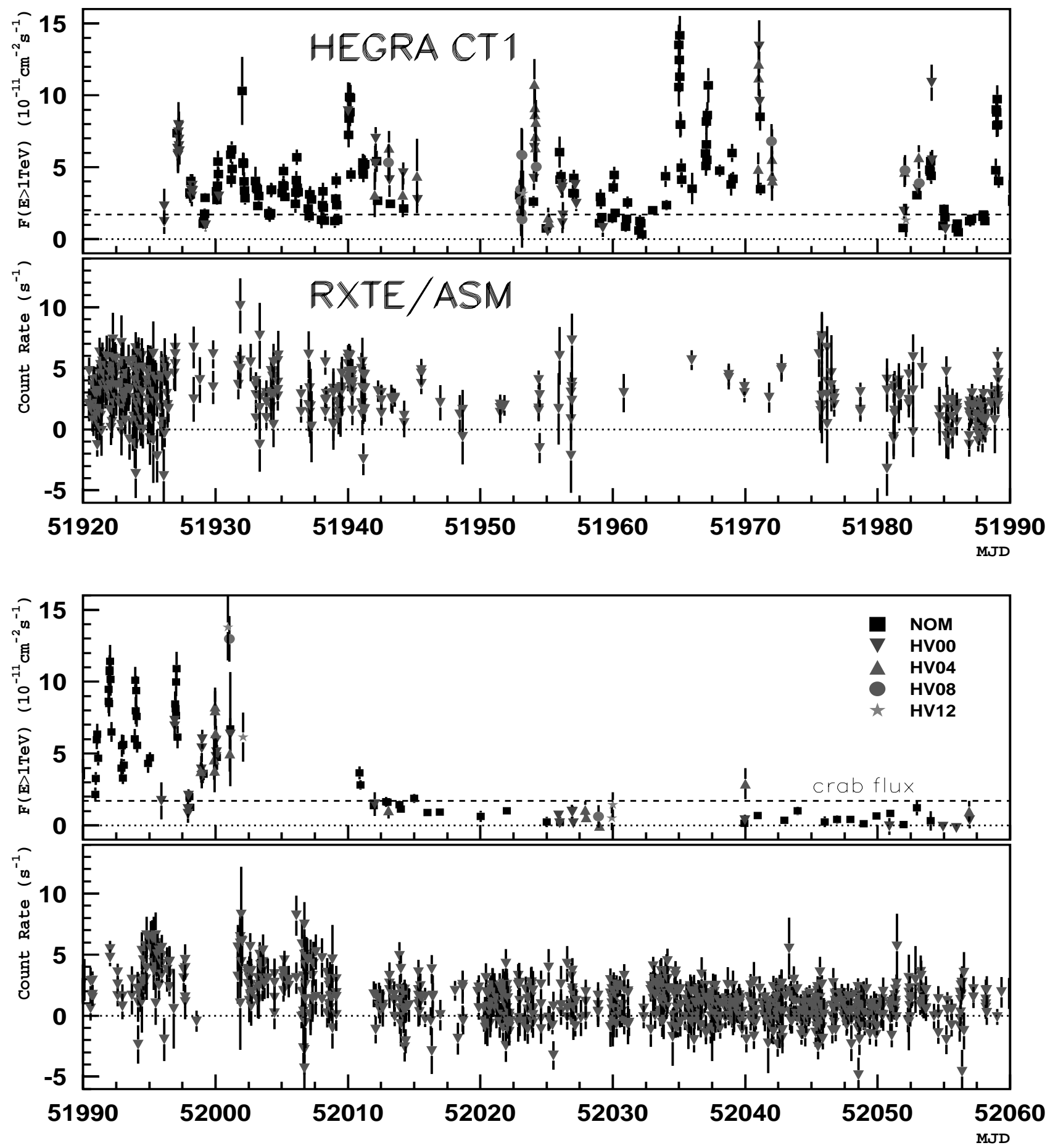

Fig. 3. Mkn 421 integral fluxes above $1 \mathrm{TeV}$ as measured with HEGRA CT1 for different HV settings (upper parts). The dashed line corresponds to the integral Crab flux as measured with CT1. The lower parts show the Mkn 421 count rate in the $2-10 \mathrm{keV}$ energy range as determined by the RXTE/ASM X-ray detector.

\section{The January to May 2001 light curve of Mkn 421}

During the last 6 years, Mkn 421 was regularly monitored by the HEGRA Cherenkov telescopes (Petry et al. 1996; Aharonian et al. 1999b; Krawczynski et al. 2001; Kestel \& The HEGRA Collaboration 2001; Aharonian et al. 2002). This data represents the most complete $\mathrm{TeV}$ coverage for this object.
Except for some short flares, the emission level of Mkn 421 was well below 1 Crab between 1995 and 1999 (Macomb et al. 1995; Gaidos et al. 1996; Buckley et al. 1996; Zweerink et al. 1997). In 2000, the mean flux level of Mkn 421 was $\sim 1 \mathrm{Crab}$ and increased to $\sim 2.5 \mathrm{Crab}$ in 2001. Figure 3 shows the CT1 integral flux curve $(>1 \mathrm{TeV})$ for the first five months of 2001 together with the X-ray light curve recorded by ASM 

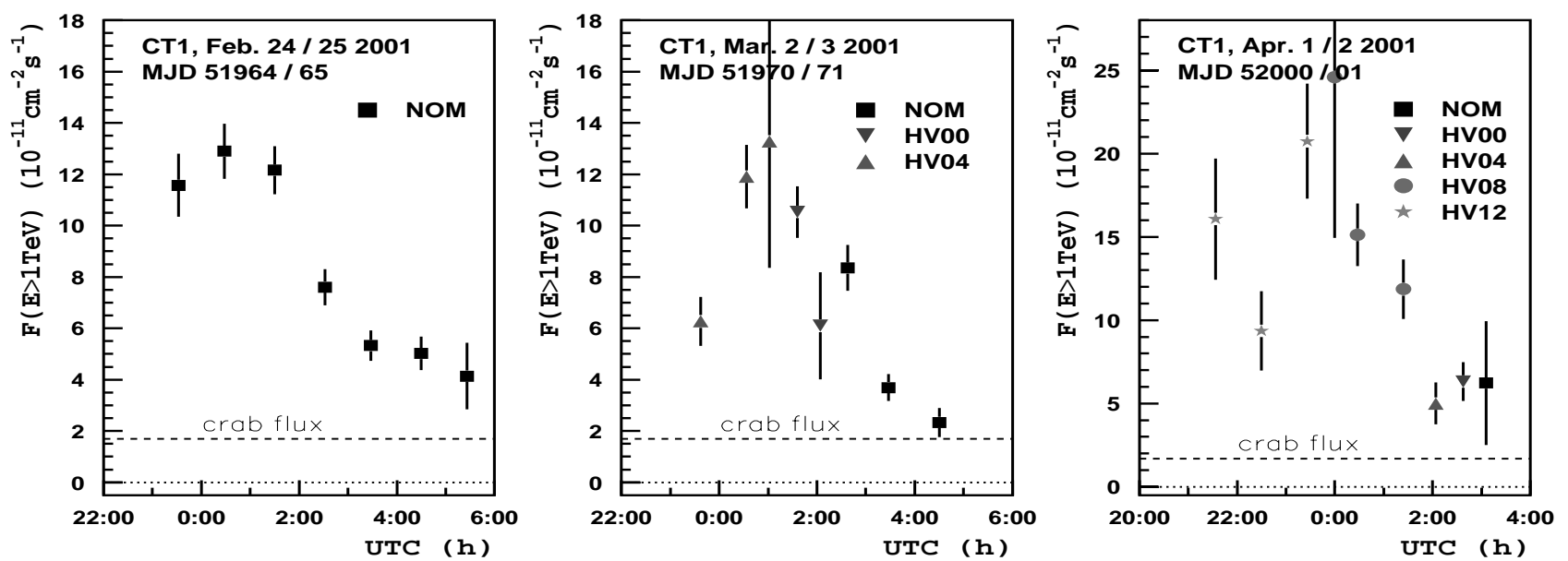

Fig. 4. Some of the strongest Mkn 421 flares in 2001.

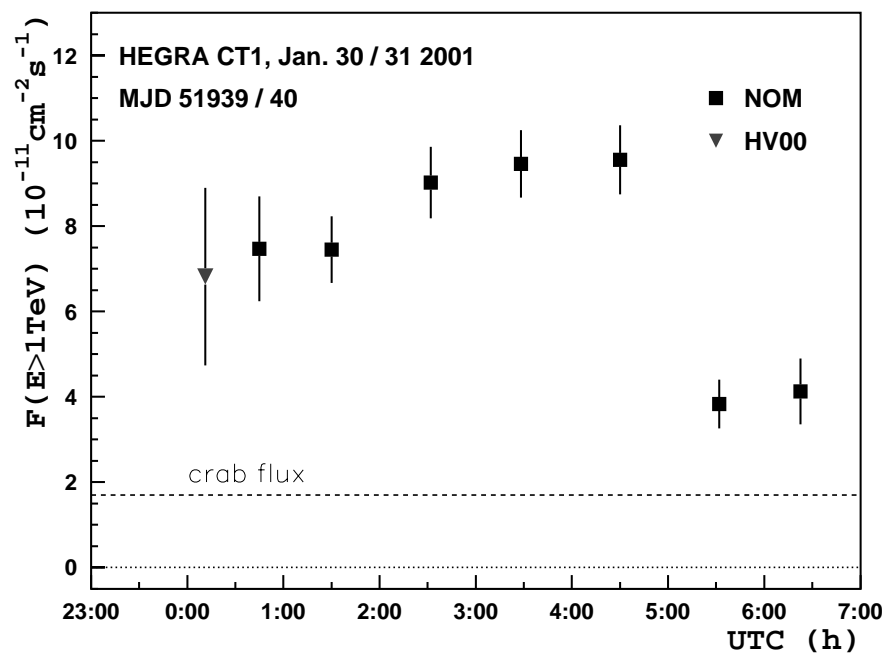

Fig. 5. Integral flux for the night of Jan. 30/31 2001 plotted in 1 hour time bins. A halving time of less than 1 hour is observed at the end of the night.

$(2-10 \mathrm{keV})$. In both the $\mathrm{TeV}$ and $\mathrm{X}$-ray range the source displays enhanced activity in the first three months of the year at an average level of $2.9 \mathrm{Crab}$ and $33 \mathrm{mCrab}$ respectively (the integral Crab flux above $1 \mathrm{TeV}$ has been derived from CT1 data as $1.7 \times 10^{-11} \mathrm{~cm}^{-2} \mathrm{~s}^{-1}$ ). As indicated in the figure, the source exhibited an average flux close to $8 \mathrm{Crab}$ for several nights. The maximum emission was observed during February 24/25, March 02/03 and April 01/02 (Fig. 4) where the emission peaked at around $10 \mathrm{Crab}$. The object activity decreased in May 2001.

Mkn 421 had been reported earlier to be highly variable on very short time scales down to $\approx 15 \min$ (Gaidos et al. 1996). New examples of intra night variability have also been found in the 2001 HEGRA dataset. Figure 5 for instance shows the $\mathrm{TeV}$ light curve for the night of January 30/31 when a flux of $\approx 5 \mathrm{Crab}$ was observed to halve in less than one hour. This variability is much faster than that observed in Mkn 501 (Aharonian et al. 1999d) and severely constrains the size of the emission region in Mkn 421.
Based on the integral fluxes above $1 \mathrm{TeV}$ (Table 4) and the "definitive product" ASM count rates ${ }^{4}$ the correlation coefficient $r$ has been calculated. We used the so-called discrete correlation function (Edelson \& Krolik 1988), which is well suited for unevenly spaced data:

$$
r(\Delta t):=\frac{\sum_{i}\left(F_{\mathrm{TeV}}\left(t_{i}\right)-\overline{F_{\mathrm{TeV}}}\right)\left(R_{\mathrm{X}}\left(t_{i}+\Delta t\right)-\overline{R_{\mathrm{X}}}\right)}{\sqrt{\sum_{i}\left(F_{\mathrm{TeV}}\left(t_{i}\right)-\overline{F_{\mathrm{TeV}}}\right)^{2} \sum_{i}\left(R_{\mathrm{X}}\left(t_{i}+\Delta t\right)-\overline{R_{\mathrm{X}}}\right)^{2}}} .
$$

Here, $\Delta t$ denotes an artificial time shift between the $\mathrm{X}$-ray count rate $R_{\mathrm{X}}$ and the TeV flux $F_{\mathrm{TeV}}$. The index $i$ runs over all coincident $\mathrm{TeV}$ and $\mathrm{X}$-ray data pairs. The error on the correlation coefficient $r(\Delta t)$ has been estimated by means of standard error propagation from Eq. (3) and its significance was derived using the Student's t-distribution on the statistics $t:=r(\Delta t) \sqrt{(N-2) /\left(1-r^{2}(\Delta t)\right)}$ as described in Press et al. (1997).

In order to take the short flaring time scales of Mkn 421 into account, we used short time bins of $1 \mathrm{~h}$ duration. In the following correlation analysis the term " $1 \mathrm{~h}$ bins" actually has a twofold meaning. First it refers to the re-binning (as weighted mean) of the flux data in $1 \mathrm{~h}$ bins. Second, only $\mathrm{TeV}$ and $\mathrm{X}$-ray data points which are separated by less than $1 \mathrm{~h}$ are used to derive the correlation coefficient.

The top panel of Fig. 6 shows $r(\Delta t)$ for $1 \mathrm{~h}$ bins and artificial time shifts of the X-ray data in units of $0.2 \mathrm{~d}$ between -20 and 20 days. The step width of $0.2 \mathrm{~d}$ corresponds to the shortest time offset, which could be resolved by the data. The X-ray and $\mathrm{TeV}$ flux variations have been found to be significantly correlated, with:

$$
r:=r(\Delta t=0)=0.83 \pm 0.09
$$

for no time shift. This correlation coefficient has a significance of $5.2 \sigma$ (based on the assumption of 26 independent data pairs). The broad peak between $-13 \mathrm{~d}$ an $13 \mathrm{~d}$ with positive correlation coefficients in the upper panel of Fig. 6 is a consequence of the long term behavior of Mkn 421 (large aver-

\footnotetext{
4 The ASM data products are provided on the web at http://heasarc.gsfc . nasa.gov/docs/xte/asm_products.html
} 

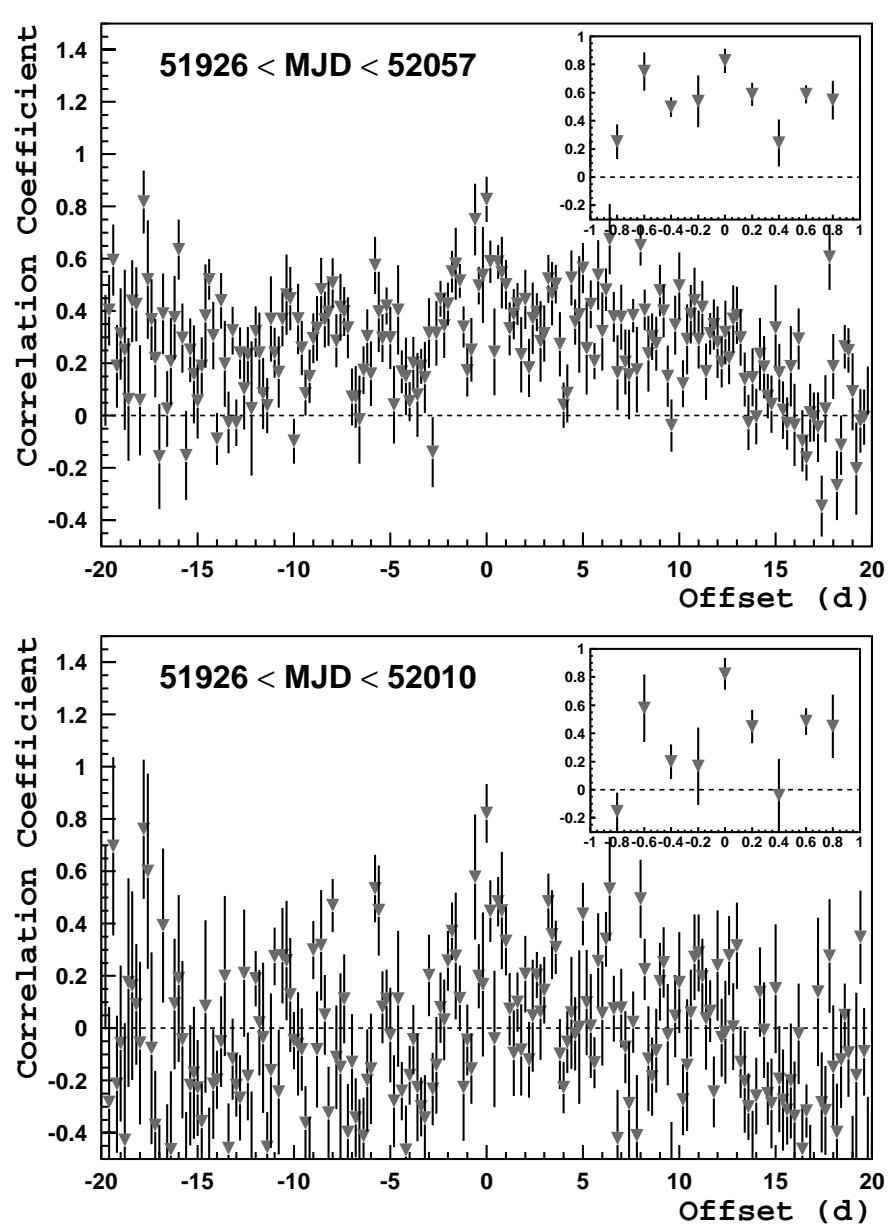

Fig. 6. The correlation coefficient between the ASM and CT1 fluxes ( $1 \mathrm{~h}$ bins) for different time offsets of the ASM data points. A maximum correlation is observed for synchronous variability of both samples (time shift shorter than $0.2 \mathrm{~d}$ ). The upper panel shows the results from the complete $\mathrm{TeV}$ and $\mathrm{X}$-ray data samples while only data up to MJD 52010 was used for the lower panel. The insets provide an expanded view of the central part.

age flux until around MJD 52010 and lower average flux afterwards, see Fig. 3) as could also be verified by means of MC simulations. If only data before MJD 52010 is used for the correlation analysis this broad peak disappears and the overall correlation becomes $r=0.82 \pm 0.12$ for 18 data pairs which corresponds to $4.2 \sigma$ (see Fig. 6 lower panel). In both cases the maximum correlation coefficient occurs at a time lag of $0 \mathrm{~d}$. The difference to the adjacent data points (see insets of Fig. 6), however, is only significant in the case of the restricted time range MJD $<52010$. Here the corresponding significances of all other data points within $\pm 1 \mathrm{~d}$ are below $2.4 \sigma$. In the case of the complete time range the adjacent data points are strongly affected by the above mentioned long term behavior of Mkn 421 and, as a result, this upper limit becomes $4.1 \sigma$.

This correlation study is affected by (a) the rapid variation of the source, (b) the limited daily observation period in IACTs, (c) the low statistics of the ASM data and the lack of full overlap in time. We have performed the same correlation analysis using larger time bins, both in ASM and CT1. The correlation coefficients for zero time lag of the ASM data can be

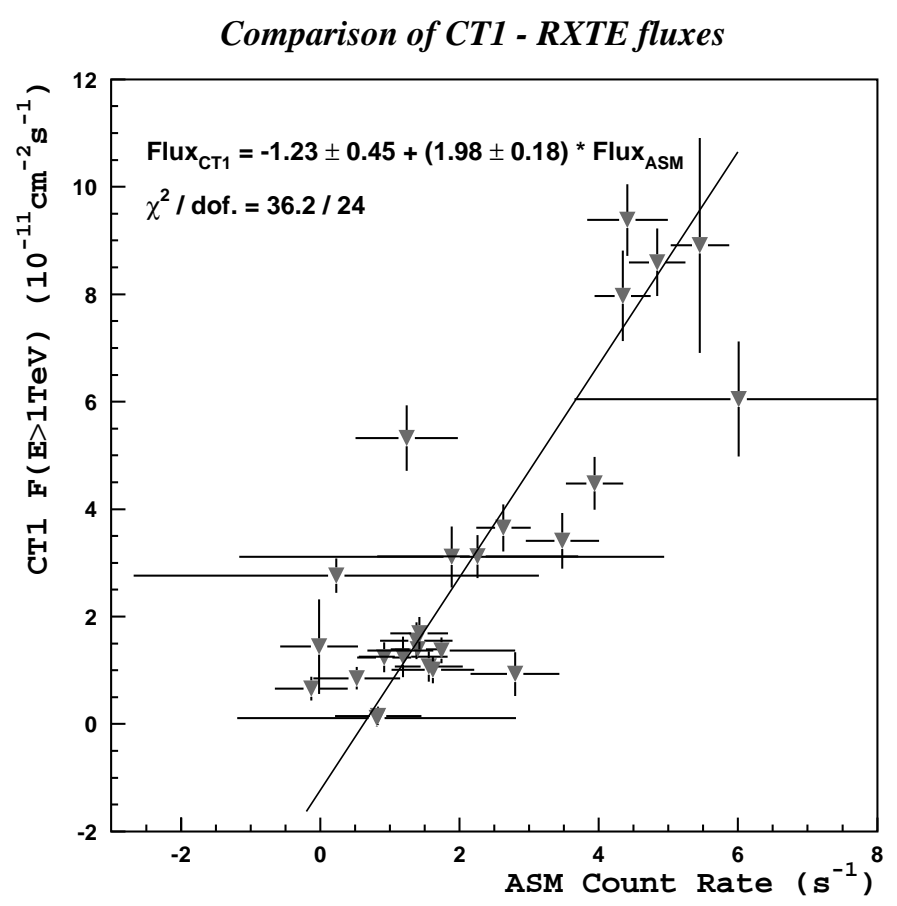

Fig. 7. Comparison of the $\mathrm{X}$-ray and $\mathrm{TeV}$ emission of Mkn 421 based on the 26 simultaneous $1 \mathrm{~h}$ bins of the 2001 data samples. The data points with the large ASM errors correspond to $1 \mathrm{~h}$ bins with only a single ASM measurement.

Table 2. Correlation coefficient of the ASM and CT1 fluxes for different sizes of the time bins. The significance of the correlation is estimated for the given number of coincident data points. No time shift was applied to the ASM fluxes.

\begin{tabular}{cccc}
\hline \hline $\begin{array}{c}\text { Bin size } \\
(\mathrm{h})\end{array}$ & $\begin{array}{c}\text { correlation } \\
\text { coefficient }\end{array}$ & $\begin{array}{c}\text { Number of } \\
\text { Data points }\end{array}$ & $\begin{array}{c}\text { Significance } \\
(\sigma)\end{array}$ \\
\hline 24 & $0.69 \pm 0.05$ & 73 & 21.2 \\
12 & $0.77 \pm 0.07$ & 52 & 18.1 \\
6 & $0.53 \pm 0.14$ & 52 & 4.2 \\
3 & $0.68 \pm 0.08$ & 38 & 4.7 \\
1 & $0.83 \pm 0.09$ & 26 & 5.2 \\
\hline
\end{tabular}

found in Table 2. Without exception, the correlation $r(\Delta t=0)$ at zero time lag was maximal or less than one sigma away from the maximum.

In order to investigate the correlation timescales we only used data before MJD 52010 since otherwise the correlation coefficients would be too much affected by the long term behavior of the Mkn 421 light curve (as mentioned above). Given this restricted time range, the maximum correlation has been found for zero time lag with a high significance of $4.2 \sigma$. For any time lag $0.2 \mathrm{~d} \leq|\Delta t| \leq 1.0 \mathrm{~d}$ the correlation coefficient was considerably smaller and less significant $(<2.4 \sigma)$. This study therefore hints to a simultaneous evolution of both light curves with an accuracy of $\leq 0.2 \mathrm{~d}$. However, the above mentioned adverse effects make any short time correlation analysis very difficult and the result has therefore also to be taken with care.

In Fig. 7 we compare the average flux values as measured by CT1 with the ASM count rate for simultaneous $1 \mathrm{~h}$ bins. 
Table 3. The results from a cutoff spectrum fit to the different dark night and moon data samples and to three different flux levels of the Mkn 421 NOM data sample. The fit parameters $F_{0}, \alpha$ and $E_{0}$ are as defined in Eq. (5); the term $\langle F(E>1 \mathrm{TeV})\rangle$ denotes the average integral flux above $1 \mathrm{TeV}$. The errors are statistical only.

\begin{tabular}{ccccccc}
\hline \hline & $\begin{array}{c}\text { Exposure } \\
\text { (h) }\end{array}$ & $\begin{array}{c}\langle F(E>1 \mathrm{TeV})\rangle \\
\left(10^{-11} \mathrm{~cm}^{-2} \mathrm{~s}^{-1}\right)\end{array}$ & $\begin{array}{c}F_{0} \\
\left(10^{-11} \mathrm{~cm}^{-2} \mathrm{~s}^{-1} \mathrm{TeV}^{-1}\right)\end{array}$ & $\alpha$ & $\begin{array}{c}\text { fixed } E_{0} \\
(\mathrm{TeV})\end{array}$ & $\chi^{2} /$ d.o.f. \\
\hline NOM & 262.7 & $4.2 \pm 0.2$ & $9.5 \pm 0.3$ & $2.39 \pm 0.04$ & 3.38 & $13.4 / 7$ \\
HV00 & 46.9 & $3.9 \pm 0.4$ & $8.3 \pm 0.4$ & $2.32 \pm 0.07$ & 3.38 & $7.0 / 6$ \\
HV04 & 23.0 & $5.4 \pm 0.6$ & $14.8 \pm 1.1$ & $3.00 \pm 0.12$ & 3.38 & $6.9 / 5$ \\
HV08 & 13.4 & $5.0 \pm 1.3$ & $16.9 \pm 3.0$ & $3.01 \pm 0.26$ & 3.38 & $2.1 / 3$ \\
HV12 & 12.5 & $5.2 \pm 1.9$ & $22.2 \pm 6.3$ & $2.77 \pm 0.31$ & 3.38 & $1.2 / 3$ \\
$0<F_{-11}<1.5^{7}$ & 65.4 & $0.8 \pm 0.1$ & $3.0 \pm 0.2$ & $2.98 \pm 0.13$ & 3.38 & $7.7 / 5$ \\
$3.0<F_{-11}<5.0$ & 56.3 & $4.0 \pm 0.1$ & $11.0 \pm 0.5$ & $2.76 \pm 0.06$ & 3.38 & $5.1 / 5$ \\
$9.0<F_{-11}$ & 14.7 & $11.8 \pm 0.7$ & $24.9 \pm 1.1$ & $2.26 \pm 0.07$ & 3.38 & $3.6 / 5$ \\
\hline$F_{-11}=F(E>1 \mathrm{TeV}) / 10^{-11} \mathrm{~cm}^{-2} \mathrm{~s}^{-1}$. & & & &
\end{tabular}

Taking into account the errors on both values, a linear fit to the data points yields:

$F_{\mathrm{CT} 1}=(-1.23 \pm 0.45)+(1.98 \pm 0.18) \times$ Rate $_{\mathrm{ASM}}$

where $^{\text {Rate }} \mathrm{ASM}_{\mathrm{A}}$ is the X-ray rate measured in counts/s and $F_{\mathrm{CT} 1}$ is the CT1 integral flux above $1 \mathrm{TeV}$ in units of $10^{-11} \mathrm{~cm}^{-2} \mathrm{~s}^{-1}$. As can be clearly seen, the linear fit agrees quite well with the data. A quadratic dependence of the TeV flux on the X-ray flux cannot be ruled out by these data. It should be noted, that the negative offset in Eq. (4) might hint towards a non-zero quiescent state in the X-ray emission of Mkn 421, similar to Mkn 501 (Aharonian et al. 1999a,d).

\section{The energy spectrum}

The method to derive energy spectra for CT1 data is described in detail in Kranich et al. (2001) where it was successfully applied to the 1997 moon and no-moon data of Mkn 501. In short, this method determines the energy of individual shower events by means of the light content and shape of the corresponding shower images. Given the energy dependent count rate, MC simulations are then used to derive the differential flux values. Effects of the finite energy resolution and the limited MC statistics are taken into account here. In an iterative process the energy spectrum is then determined from a $\chi^{2}$-fit to the differential flux values.

If the observations cover a larger ZA region the data is subdivided into several ZA bins. In order to cope with the ZA dependence of the energy threshold of an IACT, the size of the ZA bins was chosen to scale with $\cos ^{2}(\mathrm{ZA})$. The energy spectra are then determined for each ZA bin and added together by calculating the weighted mean from the differential flux values for each energy bin. For variable sources, like Mkn 421, the individual ZA bin energy spectra have to be normalized in advance ${ }^{5}$. The reference value is represented by the energy spectrum of the lowest ZA bin due to the higher statistics.

Except for some short flaring episodes Mkn 421 has shown no remarkable activity since its discovery in 1992 . The

\footnotetext{
${ }^{5}$ Since an energy spectrum at large ZAs only covers the high energy range of a low ZA spectrum, both spectra have to be at the same flux level before they can be combined.
}

Mkn 421 - Energy spectrum 2000-01

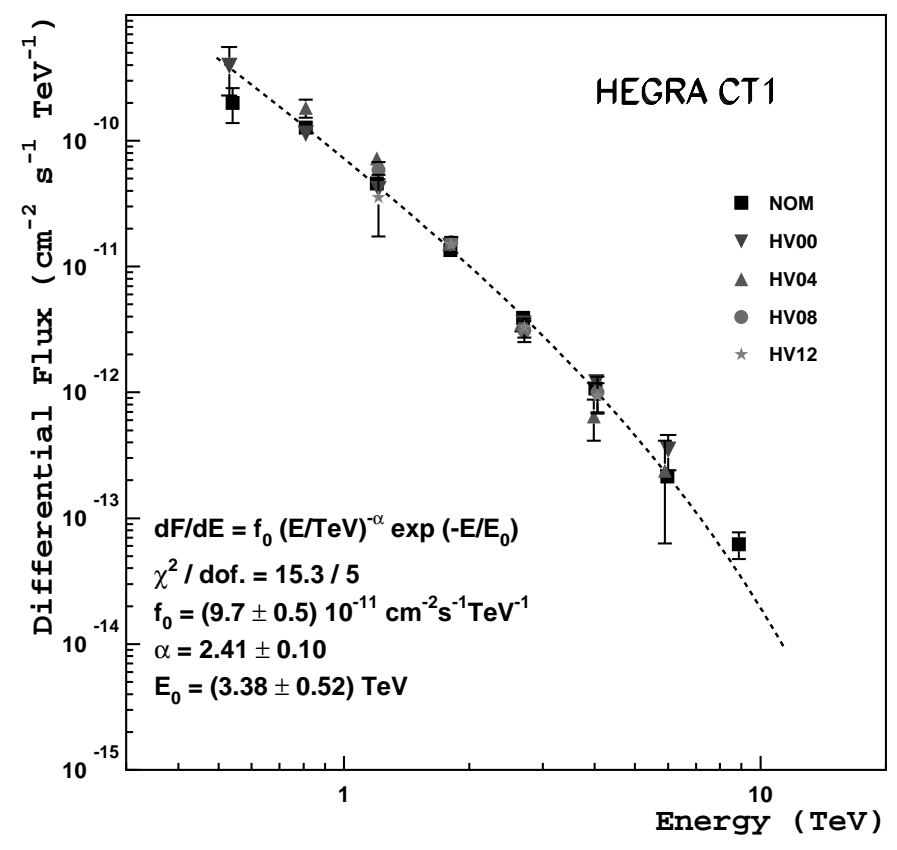

Fig. 8. The HEGRA CT1 Mkn 421 energy spectra in 2001 for the individual dark night and moon data samples (normalized at $2 \mathrm{TeV}$ ). The overall spectrum (dashed line) has been derived from the combined (as weighted mean) data points.

quiescent spectrum measured by the HEGRA CT-system in 1997 and 1998 could be acceptably fitted to a power law (Aharonian et al. 1999b) with $F_{0}=\left(12.1 \pm 0.5_{\text {stat }} \pm 4.3_{\text {syst }}\right) \times$ $10^{-12} \mathrm{~cm}^{-2} \mathrm{~s}^{-1} \mathrm{TeV}^{-1}$ and $\alpha=3.09 \pm 0.07_{\text {stat }} \pm 0.10_{\text {syst. An }}$ alternative fit to a power law with an energy cutoff could not be justified due to limited statistics. Similar results have been obtained from HEGRA CT1 in 2000 (Kestel \& The HEGRA Collaboration 2001).

The CT1 Mkn 421 energy spectrum during 2001 was determined separately for the dark night data and the different HV settings of the moon data. Due to limited statistics at large ZAs, the maximum ZA in the energy spectrum calculation was restricted to $45^{\circ}$. The results are shown in Fig. 8 and Table 3. As can be seen, there is an excellent agreement between 


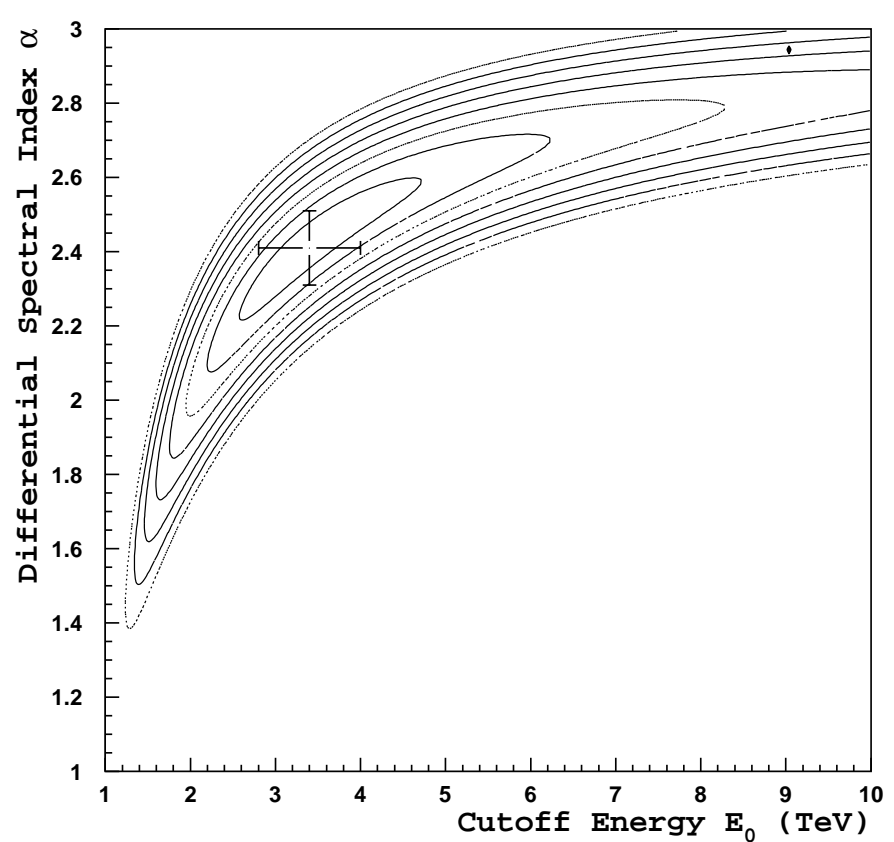

Fig. 9. The contour plot for the reduced $\chi^{2}$ values of the Mkn 421 energy spectrum fit as a function of the cutoff energy and the differential spectral index. The cross marks the result (inclusive statistical errors) from the spectral fit and the lines denote the confidence contours in $1 \sigma$ steps relative to the minimum of $2.4 \sigma$.

the individual data samples. The combined spectrum is significantly curved. A fit using a power law in the $0.5-10 \mathrm{TeV}$ energy range shows a reduced chi-square $\chi^{2} /$ d.o.f. $=56.1 / 6=9.4$, whereas a fit to a power law with an energy cutoff yields $\chi^{2} /$ d.o.f. $=15.3 / 5=3.1$. A pure power law spectrum can thus be significantly ruled out while the cutoff spectrum is still acceptable at the $2.4 \sigma$ level. The result of the second fit:

$\frac{\mathrm{d} F}{\mathrm{~d} E}=F_{0} \cdot\left(\frac{E}{\mathrm{TeV}}\right)^{-\alpha} \cdot \exp \left(-\frac{E}{E_{0}}\right)$

yields $F_{0}=\left(9.7 \pm 0.5_{\text {stat }}\right) \times 10^{-11} \mathrm{~cm}^{-2} \mathrm{~s}^{-1} \mathrm{TeV}^{-1}$, spectral index $\alpha=2.41 \pm 0.10_{\text {stat }}$ and cutoff energy $E_{0}=3.4 \pm 0.5_{\text {stat }} \mathrm{TeV}$. It should be noted that changes in $E_{0}$ are strongly correlated with changes in the spectral index $\alpha$. As can be seen from Fig. 9, $E_{0}$ is found to grow with increasing $\alpha$.

In order to estimate the systematic errors on the derived energy spectrum we repeated the complete analysis with the Photoelectron to ADC-counts conversion factor varied within its uncertainty of $18 \%$. Taking further into account the uncertainty of the effective collection areas and the correlation between the cutoff energy $E_{0}$ and the spectral index $\alpha$ - the confidence contours at the minimum $\chi^{2} /$ d.o.f. plus $1 \sigma$ were used - yields: $\alpha=2.41 \pm 0.10_{\text {stat }}\left(\begin{array}{c}+0.49 \\ -0.34\end{array}\right)_{\text {syst }}$ and $E_{0}=3.4 \pm$ $0.6_{\text {stat }}\left(\begin{array}{c}+2.8 \\ -1.1\end{array}\right)_{\text {syst }} \mathrm{TeV}$.

In order to study the spectral variations, which have been found in the HEGRA CT-system data (Aharonian et al. 2002) we derived the energy spectra from the CT1 no-moon data for three different flux levels. The fit parameters of the applied cutoff spectrum as well as some additional information is summarized in Table 3.
Mkn 421 - Energy spectrum 2000-01

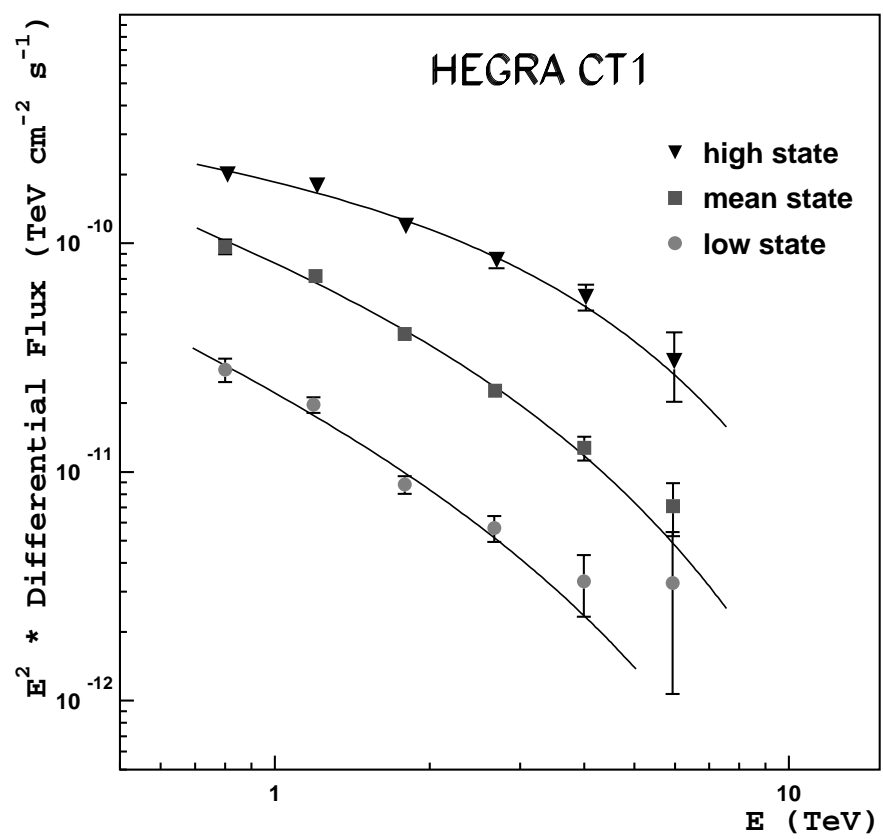

Fig. 10. The HEGRA CT1 Mkn 421 energy spectra for different emission levels. Only the CT1 no-moon data has been used here.

Using the high state spectral variables $\alpha=2.26$ and $E_{0}=$ 3.38 as fixed parameters in the fit to the mean- and low-state data a $\chi^{2} /$ d.o.f. of $57.2 / 6(\sim 9.8 \sigma)$ and $27.8 / 6(\sim 3.7 \sigma)$ respectively is obtained. Thus the spectral shape in the high-state is significantly different compared to the mean-state and lowstate data. Similarly, a comparison of the mean-state and lowstate spectrum yields a $\chi^{2} /$ d.o.f. of $7.6 / 6(\sim 0.6 \sigma)$; i.e. no significant differences could be established here. All three spectra are shown in Fig. 10 for comparison.

As can be seen from the derived spectra for the three different flux levels (Table 3) the data shows a clear flux dependence of the differential spectral index. The observed spectral hardening with increased flux level is in agreement with the results from other experiments and detectors (Aharonian et al. 2002; Krennrich et al. 2002). A closer examination shows that while the derived spectral index for the low state of Mkn 421 is similar for the CT1 $(\alpha=2.98 \pm 0.13)$ the CT system $(\alpha=3.0 \pm 0.2$, Aharonian et al. 2002) and the Whipple $(\alpha=2.72 \pm 0.11$, Krennrich et al. 2002) data, the spectral index in the mean state is significantly different $\left(\alpha_{\mathrm{CT} 1}=2.76 \pm 0.11\right.$, $\alpha_{\text {CT-system }}=2.28 \pm 0.02$ and $\alpha_{\text {Whipple }}=2.23 \pm 0.06$ ). In the case of the high state data, the agreement between the CT1 and the CT-system result is reasonably good $\left(\alpha_{\mathrm{CT} 1}=2.26 \pm 0.07\right.$ and $\alpha_{\mathrm{CT} \text {-system }}=2.06 \pm 0.03$ ) but rather poor compared to the Whipple result $\left(\alpha_{\text {Whipple }}=1.89 \pm 0.04\right)$.

Possible reasons for these discrepancies are systematic effects (the given errors are statistical only) and the different observation times covered by the three detectors. Since the spectral shape does not always change with the flux level (Aharonian et al. 2002), a different time coverage of these detectors may also lead to varying spectra for the different flux levels. 


\section{Conclusions}

Mkn 421 has been in a high state of $\mathrm{TeV} \gamma$ emission in the first months of 2001. Integral fluxes above $700 \mathrm{GeV}$ in excess of 5 Crab have been observed for several nights.

The high statistics allows one to extract a detailed light curve showing very fast variability with doubling times shorter than one hour. The time variations in the $\mathrm{TeV}$ range are significantly correlated to the $2-10 \mathrm{keV} \mathrm{X}$-ray time variations with less than $0.2 \mathrm{~d}$ time delay. This confirms earlier results on the same object (Maraschi et al. 1999; Takahashi et al. 1999).

Due to the short variability time scales of Mkn 421 and the sparse and non simultaneous data sampling of the $\mathrm{TeV}$ and $\mathrm{X}$-ray data, however, the data binning must be carefully chosen. In case of a $1 \mathrm{~d}$ binning, the main contribution to the correlation coefficient comes from the long term behavior of Mkn 421 in 2001, i.e. high emission until and reduced emission after MJD 52010. Short term variations, on the other hand, can only be probed by short data bins of about $1 \mathrm{~h}$ duration which, however, significantly reduce the number of coincident $\mathrm{TeV}$ and $\mathrm{X}$-ray data pairs.

An accurate energy spectrum up to energies of $10 \mathrm{TeV}$ could be extracted. The spectrum is compatible with a power law with differential spectral index $\alpha=2.41 \pm 0.10_{\text {stat }}\left(\begin{array}{l}+0.49 \\ -0.34\end{array}\right)_{\text {syst }}$ combined with a cutoff energy at $E_{0}=3.4 \pm 0.6_{\text {stat }}\left(\begin{array}{l}+2.8 \\ -1.1\end{array}\right)_{\text {syst }} \mathrm{TeV}$. The cutoff energy is still in agreement with the observed cutoff energy of $\sim 6 \mathrm{TeV}$ from Mkn 501 in 1997 (Aharonian et al. 1999c) and can therefore most likely be attributed to the TeV attenuation by the extragalactic background light. On the other hand, the clear differences in the energy spectrum of the two objects can be addressed to different inherent source spectra. In case the position of the inverse Compton peak is at higher energies (as for Mkn 501) one would expect a harder spectrum and, if the source spectrum is inherently curved, an increased cutoff energy. Since the synchrotron peak position has been found to increase with the emission state for both objects (Kataoka et al. 1999; Horns et al. 2001), the same mechanism can also be responsible for the flux dependent spectrum variations of Mkn 421.

As one would expect, the derived energy spectrum from Mkn 421 is in good agreement with the results from the CT-system (Aharonian et al. 2002) where the differential spectral index and the cutoff energy were determined as $\alpha=2.19 \pm$ $0.02_{\text {stat }} \pm 0.01_{\text {syst }}$ and $E_{0}=3.6\left(\begin{array}{l}+0.4 \\ -0.3\end{array}\right)_{\text {stat }}\left(\begin{array}{l}+0.9 \\ -0.8\end{array}\right)_{\text {syst }}$ respectively. It should be noted that only a small fraction of events $(\sim 10 \%)$ in the CT-system and CT1 NOM data sample are identical.

Acknowledgements. We would like to thank the IAC for the excellent working conditions on the La Palma Observatorio Roque de los Muchachos. The support of the Spanish CICYT and German BMBF is gratefully acknowledged. The ASM results were obtained through the High Energy Astrophysics Science Archive Research Center Online Service, provided by the NASA/Goddard Space Flight Center.

\section{References}

Aharonian, F., Akhperjanian, A., Beilicke, M., et al. 2002, A\&A, 393, 89

Aharonian, F., Akhperjanian, A. G., Barrio, J. A., et al. 1999a, A\&A, 349,29

Aharonian, F. A., Akhperjanian, A. G., Andronache, M., et al. 1999b, A\&A, 350, 757

Aharonian, F. A., Akhperjanian, A. G., Barrio, J. A., et al. 1999c, A\&A, 349, 11

Aharonian, F. A., Akhperjanian, A. G., Barrio, J. A., et al. 1999d, A\&A, 342, 69

Börst, H. G., Götting, N., \& Remillard, R. 2001, IAU Circ., 7568, 3

Buckley, J. H., Akerlof, C. W., Biller, S., et al. 1996, ApJ, 472, L9

Cortina, J., Barrio, J. A., Rauterberg, G., \& The HEGRA Collaboration 2000, in GeV-TeV Gamma Ray Astrophysics Workshop: towards a major atmospheric Cherenkov detector, 368

Cortina, J., Kestel, M., Kranich, D., et al. 2002, in Proc. The Universe viewed in Gamma-rays, Tokyo, to be published

Dermer, C. D., \& Schlickeiser, R. 1994, ApJS, 90, 945

Djannati-Ata1, A., Piron, F., Barrau, A., et al. 1999, A\&A, 350, 17

Edelson, R. A., \& Krolik, J. H. 1988, ApJ, 333, 646

Gaidos, J. A., Akerlof, C. W., Biller, S. D., et al. 1996, Nature, 383, 319

Gould, R. J., \& Schréder, G. 1966, Phys. Rev. Lett., 16, 252

Horns, D., Kohnle, A., Remillard, R. A., \& The HEGRA Collaboration 2001, in Proc. 27th ICRC, Hamburg, 106

Kataoka, J., Mattox, J. R., Quinn, J., et al. 1999, ApJ, 514, 138

Kestel, M. \& The HEGRA Collaboration. 2001, in Proc. 27th ICRC, Hamburg, 2634

Kranich, D. 2001, Ph.D. Thesis, Technische Universität München

Kranich, D., Kestel, M., \& The HEGRA Collaboration. 2001, in Proc. 27th ICRC, Hamburg, 2683

Kranich, D., Mirzoyan, R., Petry, D., et al. 1999, Astropart. Phys., 12, 65

Krawczynski, H., Sambruna, R., Kohnle, A., et al. 2001, ApJ, 559, 187

Krennrich, F., Bond, I. H., Bradbury, S. M., et al. 2002, ApJ, 575, L9

Lessard, R. W., Buckley, J. H., Connaughton, V., \& Le Bohec, S. 2001, Astropart. Phys., 15, 1

Macomb, D. J., Akerlof, C. W., Aller, H. D., et al. 1995, ApJ, 449, L99

Maraschi, L., Fossati, G., Tavecchio, F., et al. 1999, Astropart. Phys., 11,189

Mirzoyan, R., Kankanian, R., Sawallisch, P., et al. 1994, Nucl. Instr. Meth. Phys. Res. A, 351, 513

Okumura, K., Asahara, A., Bicknell, G. V., et al. 2002, ApJ, 579, L9

Petry, D., Bradbury, S. M., Konopelko, A., et al. 1996, A\&A, 311, L13

Press, W. H., Teukolsky, S. A., Vetterling, W. T., \& Flannery, B. P. 1997, Numerical Recipes in C, 2nd ed. (Cambridge University Press)

Punch, M., Akerlof, C. W., Cawley, M. F., et al. 1992, Nature, 358, 477

Samuelson, F. W., Biller, S. D., Bond, I. H., et al. 1998, ApJ, 501, L17 Schweizer, T. 2002, Ph.D. Thesis, Universitat Autònoma de Barcelona Sobczyńska, D., \& Lorenz, E. 2002, in Nuclear Instruments and Methods, Phys. Res. A, 490, 124

Stecker, F. W., \& de Jager, O. C. 1993, ApJ, 415, L71

Stecker, F. W., de Jager, O. C., \& Salamon, M. H. 1992, ApJ, 390, L49

Takahashi, T., Madejski, G., \& Kubo, H. 1999, Astropart. Phys., 11, 177

Zweerink, J. A., Akerlof, C. W., Biller, S. D., et al. 1997, ApJ, 490, L141 